\title{
高可见光透过、高紫外截止镀膜玻璃的制备
}

\author{
王红燕, 张浩, 姜宏, 汪国庆, 熊春荣
}

(海南大学 南海海洋资源利用国家重点实验室, 海南省特种玻璃实验室, 海口 570228)

摘 要: 为了提高镀膜玻璃的可见光透过率, 本研究通过反胶束溶液刻蚀法制备出一种新型玻璃基板, 即表面多孔 玻璃。玻璃表面形成了蜂窝状的多孔膜层，减小了对可见光的反射率，从而使可见光平均透过率提高了 $7 \%$ 。通过 一系列射频磁控溅射实验，探索了可见光平均透过率高，紫外阻隔率最高的最佳工艺条件。在此条件下，分别在制 备的表面多孔玻璃和普通玻璃上镀 $\mathrm{CeO}_{2} / \mathrm{TiO}_{2}$ 防紫外线膜, 并采用紫外-可见分光光度计、SEM、XRD、XPS 等测 试手段对样品进行了分析表征。结果表明，在相同的镀膜条件下，当镀膜后表面多孔玻璃与镀膜普通玻璃的紫外光 阻隔率均为 $99 \%$ 时, 表面多孔玻璃镀膜后的可见光平均透过率为 $85 \%$, 而普通玻璃镀膜后的可见光平均透过率仅 为 79\%。此外，玻璃表面上的孔结构还提高了薄膜与基板的接触面积，使膜基结合力提高 2 倍左右。

关 键 词: 多孔玻璃; 透过率; $\mathrm{CeO}_{2} / \mathrm{TiO}_{2}$ 薄膜; 磁控溅射

中图分类号: TQ174 文献标识码: A

\section{Preparation of Coating Glass with High Visible Transmittance and High UV Cut-off}

\author{
WANG Hong-Yan, ZHANG Hao, JIANG Hong, WANG Guo-Qing, XIONG Chun-Rong
}

(Skate Key Laboratory of Marine Resource Utilization in South China Sea, Special Glass Key Lab of Hainan Province, Hainan University, Haikou 570228, China)

\begin{abstract}
A glass with a honeycomb surface was prepared by a reverse micellar solution etching technology. The honeycomb surface might reduce the light reflection and increase the average transmittance of visible light by nearly 7\%. The chemical etching technology is environment friendly for unuse of HF or fluosilicate. To compare the visible light transmittance of the coated glass, $\mathrm{CeO}_{2} / \mathrm{TiO}_{2}$ films were deposited on the honeycomb surface glass and raw glass by radio frequency magnetron sputtering. A series of optimization experiments were carried out to obtain a coated glass with high visible transmittance and high UV cut-off. The samples were characterized by UV-Vis spectrophotometer, SEM, XRD, and XPS. When the UV cut-off ratios were 99\% for both the honeycomb surface glass and the raw glass, the average transmittance of visible light was $85 \%$ for the coated honeycomb surface glass, and only $79 \%$ for the coated raw glass prepared under the same coating conditions. In addition, the porous structure on the glass surface also improved the contact area between the film and the substrate. Therefore, the binding force between film and substrate was increased by about 2 times.
\end{abstract}

Key words: porous glass; transmittance; $\mathrm{CeO}_{2} / \mathrm{TiO}_{2}$ coating; magnetron sputtering

紫外线能导致皮肤老化，诱发皮肤癌、白内障等疾病，近年来臭氧层遭到破坏，紫外线辐射增强，

收稿日期: 2016-11-01; 收到修改稿日期：2017-01-08

基金项目：海南省重点研发计划项目(ZDYF2016017); 国家自然科学基金(51562008)

Key Research and Development Project of Hainan Province (ZDYF2016017); National Natural Science Foundation of China (51562008)

作者简介：王红燕(1987-)，女，硕士研究生. E-mail: why8987@foxmail.com

通讯作者：熊春荣，教授. E-mail: 1192537479@qq.com 
影响到人们的日常生活。目前, 防紫外线镀膜玻璃 在越来越多的领域获得了应用。但是防紫外线膜也 会降低可见光的透过, 通常当膜的紫外截止率达 $98 \%$ 以上时, 可见光透过率通常被降低到 $75 \%{ }^{[1]}$, 因此, 在对可见光透过率要求高的领域, 如建筑、汽 车制造及某些电子领域, 可见光透过率低的防紫外 玻璃的应用受到一定影响 ${ }^{[2-3]}$ 。

$\mathrm{CeO}_{2} / \mathrm{TiO}_{2}$ 薄膜具有很好的紫外吸收性能, Sainz ${ }^{[4]}$ 和 Morimoto ${ }^{[5]}$ 等首先采用氯化铈和不同种类 的醇盐制备 $\mathrm{CeO}_{2} / \mathrm{TiO}_{2}$ 溶胶, 用提拉法制备 $\mathrm{CeO}_{2} / \mathrm{TiO}_{2}$ 膜层, 但是通过溶胶一凝胶法制备的薄膜 结合力不好, 容易脱落 ${ }^{[4-5]}$ 。绍明迪等 ${ }^{[6]}$ 在浮法玻璃 配方中加入 $\mathrm{CeO}_{2}$, 对紫外线有一定的屏蔽能力, 紫 外截止率为 $92 \%$ 左右, 效果不如磁控溅射方法好。 赵青南等 ${ }^{[7-9]}$ 分别以纯 $\mathrm{CeO}_{2}$ 与纯 $\mathrm{TiO}_{2}$ 为靶材以及 $\mathrm{CeO}_{2} / \mathrm{TiO}_{2}$ 混合氧化物为靶材, 采用磁控溅射制备 了 $\mathrm{CeO}_{2} / \mathrm{TiO}_{2}$ 膜, 通过优化溅射的工艺条件, 紫外 截止率高达 $99 \%$, 可见光最大透过率达 $79 \%$ 。

本工作采用表面活性剂包裹化学蚀刻液形成微 球, 先对玻璃表面进行蚀刻, 形成纳米孔, 提高玻 璃本体的可见光透过率 ${ }^{[10-11]}$, 然后再采用射频磁控 溅射法在玻璃表面镀制 $\mathrm{CeO}_{2} / \mathrm{TiO}_{2}$ 膜, 从而获得了 高可见光透过、高紫外截止镀膜玻璃。

\section{1 实验方法}

\section{1 表面多孔玻璃的制备}

将 $0.5 \mathrm{~g}$ CTAB、 $30 \mathrm{~mL}$ 正庚烷及 $10 \mathrm{~mL}$ 正辛醇 搅拌均匀, 然后加入 $10 \mathrm{~mL}(2 \mathrm{~mol} / \mathrm{L})$ 的硫酸, 形成 反胶束刻蚀溶液。然后在反胶束刻蚀溶液中放入先 后经去离子水、丙酮及无水乙醇超声清洗净的钠钻 硅普通玻璃原片, 在烘箱中 $100^{\circ} \mathrm{C}$ 处理 $3 \mathrm{~h}$ 。再使用 蒸馏水洗干净, 吹干, 制得高可见光透过率的表面 多孔玻璃。

\section{2 磁控溅射镀膜}

利用 K14-439 型单室高真空三靶磁控溅射镀膜 机在玻璃基片上射频磁控溅射制备薄膜。基片尺寸 为 $76 \mathrm{~mm} \times 25 \mathrm{~mm} \times 1 \mathrm{~mm}$, 基片先经过去离子水超声 清洗 $5 \mathrm{~min}$, 然后在 $60^{\circ} \mathrm{C}$ 丙酮溶液中超声清洗 $10 \mathrm{~min}$, 再在去离子水中超声清洗 $5 \mathrm{~min}$, 用氮气枪吹干, 备 用。实验所用的靶材为 $\mathrm{CeO}_{2}$ 和 $\mathrm{TiO}_{2}$ 陶瓷靶, 直径 $10 \mathrm{~cm}$, 其纯度均为 99.99\%。使用前 $\mathrm{CeO}_{2}$ 和 $\mathrm{TiO}_{2}$ 靶材经去离子水超声清洗 $5 \mathrm{~min}$, 然后在 $60^{\circ} \mathrm{C}$ 丙酮 溶液中超声清洗 $10 \mathrm{~min}$, 再放入去离子水中超声清 洗 $5 \mathrm{~min}$, 用氮气枪吹干, 备用。当镀膜腔室真空为
$2.0 \times 10^{-3} \mathrm{~Pa}$ 时, 通入氧气及氩气。溅射时, 氩气(纯 度 99. 999\%) 流量为 $50 \mathrm{sccm}$, 氧气(纯度 99.99\%) 流量为 $1 \mathrm{sccm}$, 通过调节气缸调节阀使工作气压保持 在 $0.8 \mathrm{~Pa}$; 基片温度为 $313 \mathrm{~K}$, 样品台转速为 $10 \mathrm{r} / \mathrm{min}$, 靶基距是 $80 \mathrm{~mm}$, 预溅射 $15 \mathrm{~min}$ 后开始镀膜。

\section{3 表征及性能测试}

采用 D/max-RA 型转靶 X 射线衍射(XRD)仪进 行样品的物相分析, 测试条件为 $\mathrm{Cu}$ 靶, $\mathrm{K} \alpha$ 射线, 加速 电压为 $40 \mathrm{kV}$, 扫描速度为 $4 \% \mathrm{~min}$ 。采用 TESCAN 公 司 MIRA3LMH/LMU 型扫描电镜(SEM)对样品表面 和断面的形貌进行分析。采用 VG-Scientific ESCALAB MK II 分析仪测试样品的 X 射线光电子能谱 (XPS), $\mathrm{Mg} \mathrm{K \alpha}$ 为辐射源, 以 $\mathrm{C} 1(284.6 \mathrm{~V})$ 作为参比。 采用 PerkinElmer 公司生产的 Lambda 35 型紫外-可 见分光光度计测量镀膜玻璃的可见光与紫外的透过 率。采用 Bruker 公司生产的 Dektak XT 的台阶仪 来测量镀膜玻璃的膜层厚度。选用 Bruker 公司生 产的纳米力学综合测试系统测试薄膜的划痕性能。

\section{2 结果与讨论}

\section{1 表面多孔玻璃}

通过表面活性剂包裹的反胶束化学刻蚀微球处 理的玻璃，其表面及侧面形貌见图 1 所示。由图 1(a) 可见, 玻璃表面形成了比较均匀的孔洞结构, 直径 约为 $20 \mathrm{~nm}$, 刻蚀深度约为 $150 \mathrm{~nm}$ (图 2(b))。根据 文献[12-13]可知，通过化学腐蚀的办法在基底表面 形成一层凸凹不平的具有 “蜂眼效应” 的多孔膜层, 使这层的折射率系数发生了改变, 加上凹坑和凸起 使入射光线发生较多的漫反射，抑制因镜面反射损 失的光，从而达到减反射的目的。

通过分光光度计测量了玻璃原片与化学刻蚀后 玻璃的透光率(图 2), 化学蚀刻后, 可见光平均透过 率达 98\%以上，相对于玻璃原片提高了 7\%。在阳 光下, 把两种玻璃放在草坪上拍照(图 3), 玻璃原片 由于光反射强, 呈现白茫茫的表面; 而表面多孔玻 璃由于减少了光反射, 太阳光基本都透过, 只能从 玻璃的边沿可视玻璃的存在。

目前国内外主要是在玻璃表面镀减反射膜，利 用膜之间不同的折射率对反射光的干涉原理来达到 减少反射的效果。溶胶一凝胶技术和磁控溅射法是我 国现在使用较为成熟的运用离线法镀减反膜的工艺, 但这两种玻璃减反增透镀膜的耐磨性能差、寿命不 长 $^{[14]}$; 膜间容易出现离子渗透, 减反增透的效果不 断降低。另外，磁控溅射镀膜，设备投资大，维修费 

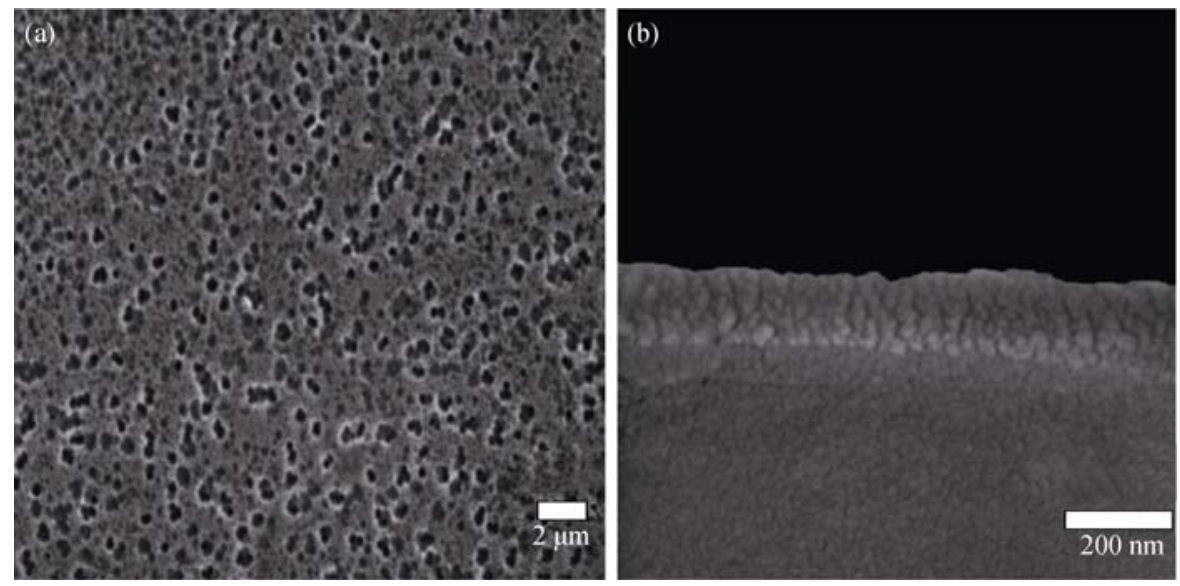

图 1 表面多孔玻璃的(a)表面和(b)断面的 SEM 照片

Fig. 1 Top view (a) and cross section (b) SEM images of the porous glass

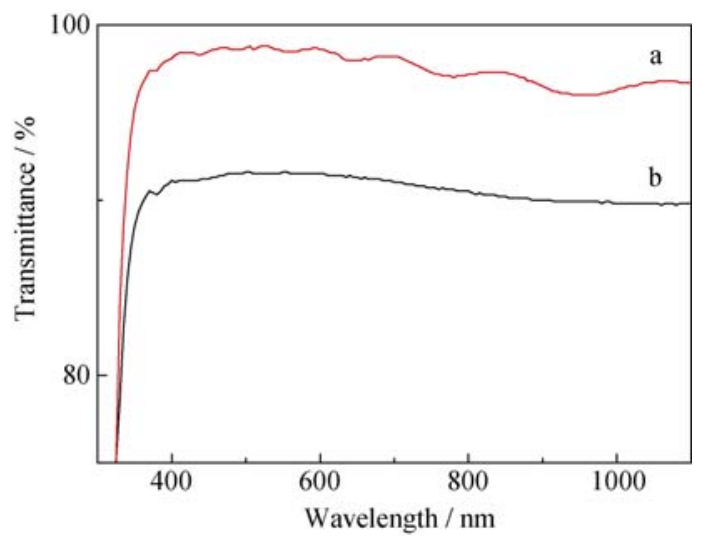

图 2 表面多孔玻璃(a)与玻璃原片(b)的透过率曲线

Fig. 2 Transmittances of the honeycomb surface glass (a) and the raw glass (b)

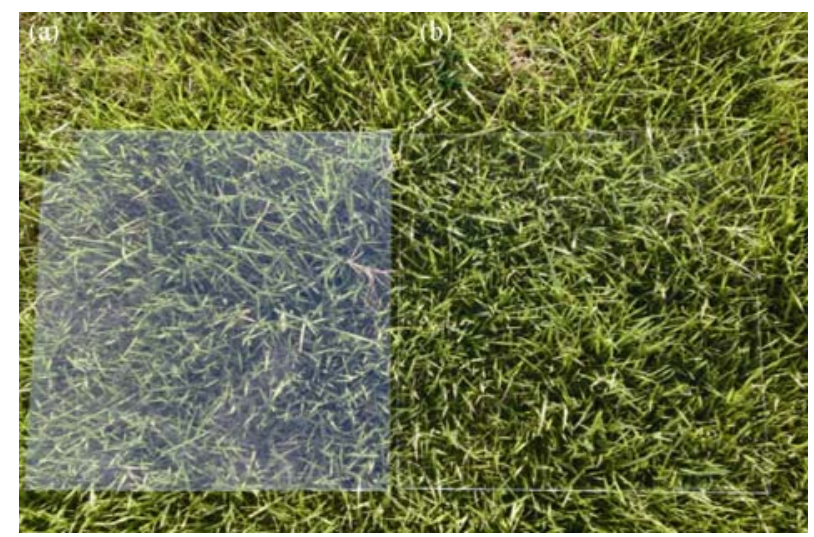

图 3 阳光下玻璃原片(a)和表面多孔玻璃(b)的数码照片

Fig. 3 Digital photos of the raw glass (a) and the honeycomb surface glass (b) under sunlight

用与生产成本高。而本研究通过反胶束化学微球直 接在玻璃本体上刻蚀处理，不仅投入低、能耗低，而 且避免了薄膜附着力差、热稳定性差等问题, 这种 减反也是玻璃增透的一种重要发展趋势 ${ }^{[15-16]}$ 。

\section{2 磁控浌射镀 $\mathrm{CeO}_{2} / \mathrm{TiO}_{2}$ 膜}

\subsection{1 不同溅射功率对薄膜性能的影响}

以玻璃原片为基片，初步考察了溅射功率对膜 紫外截止和可见光透过性能的影响，镀膜时间均为 $60 \mathrm{~min}, \mathrm{TiO}_{2}$ 靶的溅射功率固定为 $140 \mathrm{~W}$, 通过改变 $\mathrm{CeO}_{2}$ 靶的溅射功率, 制备了 $\mathrm{A} 1 \sim \mathrm{A} 6$ 六个样品(表 1, 图 4)。使用布鲁克台阶仪测出样品的膜厚，薄膜厚

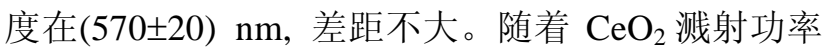
的增加, 可见光平均透过率逐渐减小, 紫外光截止 率先增大后减小, $\mathrm{CeO}_{2}$ 与 $\mathrm{TiO}_{2}$ 的功率分别为 $120 \mathrm{~W}$ 和 $140 \mathrm{~W}$ 时，紫外光截止率为 $99.1 \%$, 可见光平均 透过率为 $76.9 \%$, 相对性能较好。溅射过程中 Ce 比 $\mathrm{Ti}$ 更容易溅射 ${ }^{[1]}$, 从表 1 可以得知, 当 $\mathrm{CeO}_{2}$ 与 $\mathrm{TiO}_{2}$ 的溅射功率分别为 $120 \mathrm{~W} 、 140 \mathrm{~W}$ 时, 膜中 $\mathrm{Ce} / \mathrm{Ti}$ 原子比为 $50 \%$, 紫外截止性能最优。样品 $\mathrm{A} 1 \sim \mathrm{A} 3$, 随着 $\mathrm{CeO}_{2}$ 溅射功率的增加, 吸收边波长和截止波 长向长波方向移动(图 5), 吸收边波长从 $420 \mathrm{~nm}$ 增大 到 $475 \mathrm{~nm}$, 截止波长从 $350 \mathrm{~nm}$ 增大到 $380 \mathrm{~nm}$; 样品 $\mathrm{A} 4 \sim \mathrm{A6}$, 随着 $\mathrm{CeO}_{2}$ 溅射功率的增加, 吸收边波长和 截止波长向短波方向移动, 吸收边波长从 $460 \mathrm{~nm}$ 减 小到 $425 \mathrm{~nm}$, 截止波长从 $370 \mathrm{~nm}$ 减小到 $340 \mathrm{~nm}$ 。所 以样品 A3 的紫外光截止率达到最大值 ${ }^{[1]}$ 。

改变 $\mathrm{CeO}_{2}$ 溅射功率，薄膜中 $\mathrm{Ce} 、 \mathrm{Ti}$ 元素的价 态也会受影响。为了进一步确定 $\mathrm{Ce} 、 \mathrm{Ti}$ 元素在薄 膜中的存在形态, 对上述薄膜进行 XPS 分析, 如图 5 所示。图 5(a)为薄膜的 Ti2p 电子的 XPS 图, $\mathrm{Ti}^{4+}$ 的 $T i 2 \mathrm{p}_{3 / 2}$ 及 $\mathrm{Ti} 2 \mathrm{p}_{1 / 2}$ 电子结合能分别为 $\mathrm{x}^{2}(460.46 \mathrm{eV})$ 及 $\mathrm{y}^{2}(465.9 \mathrm{eV})^{[17]} ; \mathrm{Ti}^{3+}$ 的 $\mathrm{Ti}_{2} \mathrm{p}_{3 / 2}$ 及 $\mathrm{Ti} 2 \mathrm{p}_{1 / 2}$ 的电子结 合能分别 $x^{1}(458.52 \mathrm{eV})$ 及 $\mathrm{y}^{1}(464.02 \mathrm{eV})^{[18-19]}$ 。图 5 (b) 为薄膜 Ce3d 电子的 XPS 图谱, 样品在 $\mathrm{v}^{1}(901.96 \mathrm{eV})$, $v^{2}(906.49 \mathrm{eV})$ 和 $v^{3}(918.27 \mathrm{eV})$ 处的三个峰对应 $\mathrm{Ce}^{4+}$ 的 
表 1 不同镀膜功率对 $\mathrm{CeO}_{2} / \mathrm{TiO}_{2}$ 薄膜透过率及组成的影响

Table 1 Effect of relative sputtering powers on properties and compositions of $\mathrm{CeO}_{2} / \mathrm{TiO}_{2}$ films

\begin{tabular}{ccccccc}
\hline & A1 & A2 & A3 & A4 & A5 & A6 \\
\hline Power $\left(\mathrm{TiO}_{2}\right) / \mathrm{W}$ & 140 & 140 & 140 & 140 & 140 & 140 \\
Power $\left(\mathrm{CeO}_{2}\right) / \mathrm{W}$ & 80 & 100 & 120 & 140 & 160 & 180 \\
Transmittance of visible/\% & 79.9 & 77.9 & 76.9 & 75.3 & 72.5 & 67.8 \\
UV cut-off ratios/\% & 96.8 & 97.2 & 99.1 & 98.4 & 95.3 & 89.5 \\
$(\mathrm{Ce} / \mathrm{Ti}) / \%$ & 42 & 46 & 50 & 55 & 62 & 65 \\
{$\left[\mathrm{Ce}^{3+} /\left(\mathrm{Ce}^{3+}+\mathrm{Ce}^{4+}\right)\right] / \%$} & 68 & 70 & 72 & 69 & 64 & 61 \\
{$\left[\mathrm{Ti}^{3+} /\left(\mathrm{Ti}^{3+}+\mathrm{Ti}^{4+}\right)\right] / \%$} & 57 & 59 & 64 & 58 & 56 & 55 \\
Thickness of the film/nm $^{540}$ & 550 & 570 & 580 & 587 & 595
\end{tabular}

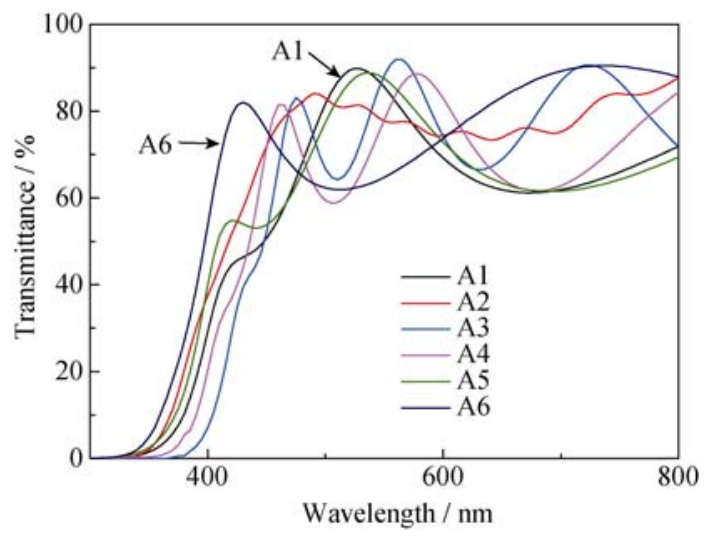

图 4 不同功率对 $\mathrm{CeO}_{2} / \mathrm{TiO}_{2}$ 薄膜透过性能的影响

Fig. 4 Influence on sputtering powers on the transmittance of the $\mathrm{CeO}_{2}-\mathrm{TiO}_{2}$ films

Ce3d d $_{3 / 2}$ 轨道的多重䢃裂峰, $u^{1}(883.46 \mathrm{eV}) 、 \mathrm{u}^{2}(887.99 \mathrm{eV})$ 和 $\mathrm{u}^{3}(899.77 \mathrm{eV})$ 对应 $\mathrm{Ce} 3 \mathrm{~d}_{5 / 2}$ 轨道电子峰。在 $\mathrm{u}^{\prime}(904.99 \mathrm{eV})$ 处对应 $\mathrm{Ce}^{3+}$ 的 $\mathrm{Ce}^{3} \mathrm{~d}_{3 / 2}$ 轨道电子峰, 在 u'(886.49 eV)处对应 Ce $3 d_{5 / 2}$ 轨道电子峰。各样品中 $\mathrm{Ce} 3 \mathrm{~d}_{3 / 2}$ 轨道与 $\mathrm{Ce} 3 \mathrm{~d}_{5 / 2}$ 轨道的能量差均为 $18.5 \mathrm{eV}$ 。 谱峰经拟合后得到 $\mathrm{Ce} 、 \mathrm{Ti}$ 元素的价态比(表 1)。随 着 $\mathrm{CeO}_{2}$ 溅射功率的提高, $\mathrm{Ce} / \mathrm{Ti} 、 \mathrm{Ce}^{3+} /\left(\mathrm{Ce}^{3+}+\mathrm{Ce}^{4+}\right)$ 和 $\mathrm{Ti}^{3+} /\left(\mathrm{Ti}^{3+}+\mathrm{Ti}^{4+}\right)$ 的比先增大后减小，与紫外截止 率的变化趋势一致, 这是由于随着 $\mathrm{Ti}^{3+}$ 含量增加, $\mathrm{Ce}^{3+}$ 含量增加, 可以提高紫外吸收率, 故在 $\mathrm{A} 3$ 处紫 外截止率达到最大。但是由于 $\mathrm{Ce}^{4+}$ 的 $4 \mathrm{f}$ 轨道己全空, 比较稳定 ${ }^{[20]}$, 一般情况下不会还原成 $\mathrm{Ce}^{3+}$, 而 $\mathrm{TiO}_{2}$

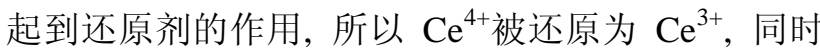
生成的 $\mathrm{Ce}^{3+}$ 吸收紫外线发生跃迁生成 $\mathrm{Ce}^{4+}$, 因此紫 外吸收强度大大增强。从图 5(c)还可以看出, 从 $\mathrm{A} 1 \sim \mathrm{A} 3$ 中可以发现 u'(886.49 eV) 处纵坐标变大, 说明 $\mathrm{Ce}-\mathrm{O}-\mathrm{Ti}$ 键逐渐增多, 这也是紫外吸收强度增大的原 因 ${ }^{[21-23]}$ 。从表 1 还可发现 A1 A5 的可见光平均透过 率逐渐降低, 这是由于 $\mathrm{CeO}_{2}$ 溅射功率增大, 在相同时 间下膜厚逐渐增厚, 从而使可见光平均透过率降低。
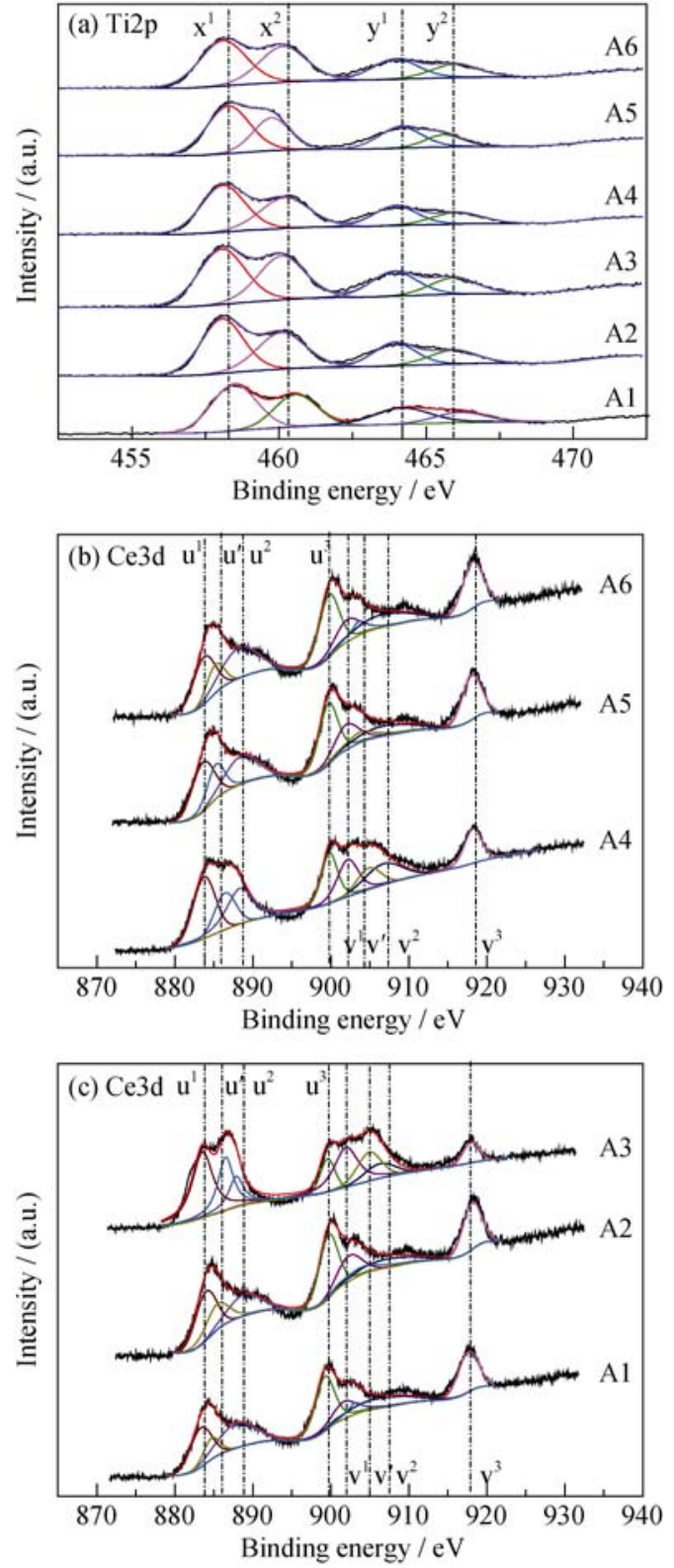

图 5 不同 $\mathrm{CeO}_{2} / \mathrm{TiO}_{2}$ 溅射功率时薄膜的 XPS 图谱

Fig. 5 XPS spectra of the $\mathrm{CeO}_{2} / \mathrm{TiO}_{2}$ films deposited under different sputtering powers

图 6 为 $\mathrm{CeO}_{2}$ 靶不同溅射功率下制备的 $\mathrm{CeO}_{2} /$ $\mathrm{TiO}_{2}$ 薄膜的 XRD 图谱。A1 和 A2 的衍射峰为锐钛 矿 $\mathrm{TiO}_{2}$ 的特征峰, 这是由于 $\mathrm{CeO}_{2}$ 溅射功率较小, $\mathrm{TiO}_{2}$ 为主要成分。增大 $\mathrm{CeO}_{2}$ 溅射功率, $\mathrm{A} 3$ 和 $\mathrm{A} 4$ 的 衍射峰逐渐宽化, 强度减小, 且在 $2 \theta=25.1^{\circ}$ 处的波 包峰右移至 $2 \theta=30^{\circ}$ [24]。然而随着 $\mathrm{CeO}_{2}$ 溅射功率的 进一步增加, A4 和 A5 的波包峰左移至 $2 \theta=28^{\circ}$ 处, 这是因为 $\mathrm{CeO}_{2}$ 和 $\mathrm{Ce}_{2} \mathrm{O}_{3}$ 的特征衍射峰分别位于 $2 \theta=28.5^{\circ}$ 及 $30.2^{\circ}$ 处, 增加 $\mathrm{CeO}_{2}$ 溅射功率, $\mathrm{Ce}^{4+}$ 的相 对含量增多，这与文献报道一致 ${ }^{[25-26]}$ 。

\subsection{2 膜厚对薄膜的性能的影响}

以玻璃原片为基板，当 $\mathrm{TiO}_{2} 、 \mathrm{CeO}_{2}$ 靶的溅射功 


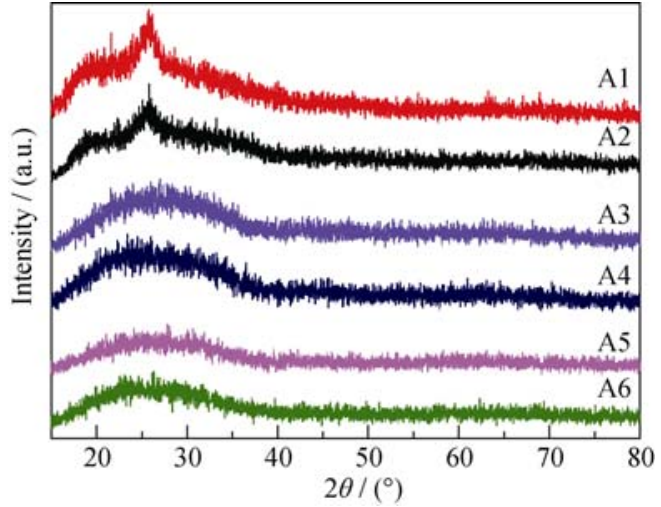

图 6 不同溅射功率制备的 $\mathrm{CeO}_{2} / \mathrm{TiO}_{2}$ 薄膜的 XRD 图谱 Fig. 6 XRD patterns of the $\mathrm{CeO}_{2} / \mathrm{TiO}_{2}$ films deposited under different sputtering powers

率分别为 $140 \mathrm{~W} 、 120 \mathrm{~W}$ 时, 改变镀膜时间研究薄 膜厚度对结构与性能的影响。从表 2 可以看出, 溅 射时间从 $10 \mathrm{~min}$ 延长到 $50 \mathrm{~min}$, 薄膜由 $127.53 \mathrm{~nm}$ 增大到 $554.87 \mathrm{~nm}$ 。从薄膜的切面 SEM 照片(图 7) 也可直接观察到膜厚度的增加。

随着薄膜厚度增加, 吸收边波长由 $425 \mathrm{~nm}$ 增大 到 $460 \mathrm{~nm}$, 截止波长由 $315 \mathrm{~nm}$ 增大到 $375 \mathrm{~nm}$ (图 8), 虽然有效提高了紫外光的截止率, 但可见光的透过 性不断下降。当镀膜时间为 $10 \mathrm{~min}$ 时, 紫外光的截 止率为 $79.8 \%$, 可见光平均透过率为 $86.4 \%$; 当镀膜 时间为 $50 \mathrm{~min}$ 时, 紫外光的截止率达 $99.2 \%$, 但可 见光平均透过率降至 $70.1 \%$ 左右。镀膜时间长有利 于薄膜防紫外的性能, 但是为了获得尽可能高的可
表 2 镀膜时间对 $\mathrm{CeO}_{2} / \mathrm{TiO}_{2}$ 薄膜透过率的影响

Table 2 Influence of sputtering time on the transmittance of the $\mathrm{CeO}_{2} / \mathrm{TiO}_{2}$ thin-films

\begin{tabular}{cccccc}
\hline Samples & B1 & B2 & B3 & B4 & B5 \\
\hline Sputtering time/min & 10 & 20 & 30 & 40 & 50 \\
Transmittance of visible/\% & 86.4 & 82.5 & 80.6 & 79.5 & 70.1 \\
UV cut-off ratio/\% & 79.8 & 82.2 & 88.5 & 96.6 & 99.2 \\
Thickness of the film/nm & 127 & 235 & 352 & 456 & 554 \\
\hline
\end{tabular}

见光的平均透过率，实验选取镀膜时间为 $40 \mathrm{~min}$, 并通过对玻璃基片进行化学刻蚀来提高可见光的 透过率。

\subsection{3 多孔增透玻璃对薄膜性能的影响}

优化在玻璃原片上的镀膜工艺: $\mathrm{TiO}_{2}$ 和 $\mathrm{CeO}_{2}$ 靶的 淺射功率分别为 $140 \mathrm{~W}$ 及 $120 \mathrm{~W}$, 镀膜时间为 $40 \mathrm{~min}$, 氩气流量为 $50 \mathrm{sccm}$, 氧气流量为 $1 \mathrm{sccm}$, 通过调节 气缸调节阀使工作气压保持在 $0.8 \mathrm{~Pa}$; 基片温度为 $313 \mathrm{~K}$, 样品台转速为 $10 \mathrm{r} / \mathrm{min}$, 同时对化学刻蚀后 的多孔增透玻璃和玻璃原片进行浌射镀膜, 研究了 玻璃上的多孔结构对薄膜性能的影响。在相同条件 下，表面多孔镀膜玻璃的可见光平均透过率为 $84.7 \%$, 原片镀膜玻璃的可见光平均透过率为 $79.8 \%$, 紫外光截止率均为 $99.7 \%$ (表 3)。在紫外光截止率接 近 100\%的情况下，多孔增透玻璃基片镀膜后可见 光平均透过率提高 $5 \%$ 。

通过划痕法测量了两种玻璃基片上的膜基结合 力(图 9)。从图 9(a)中可以看出玻璃原片上薄膜的结
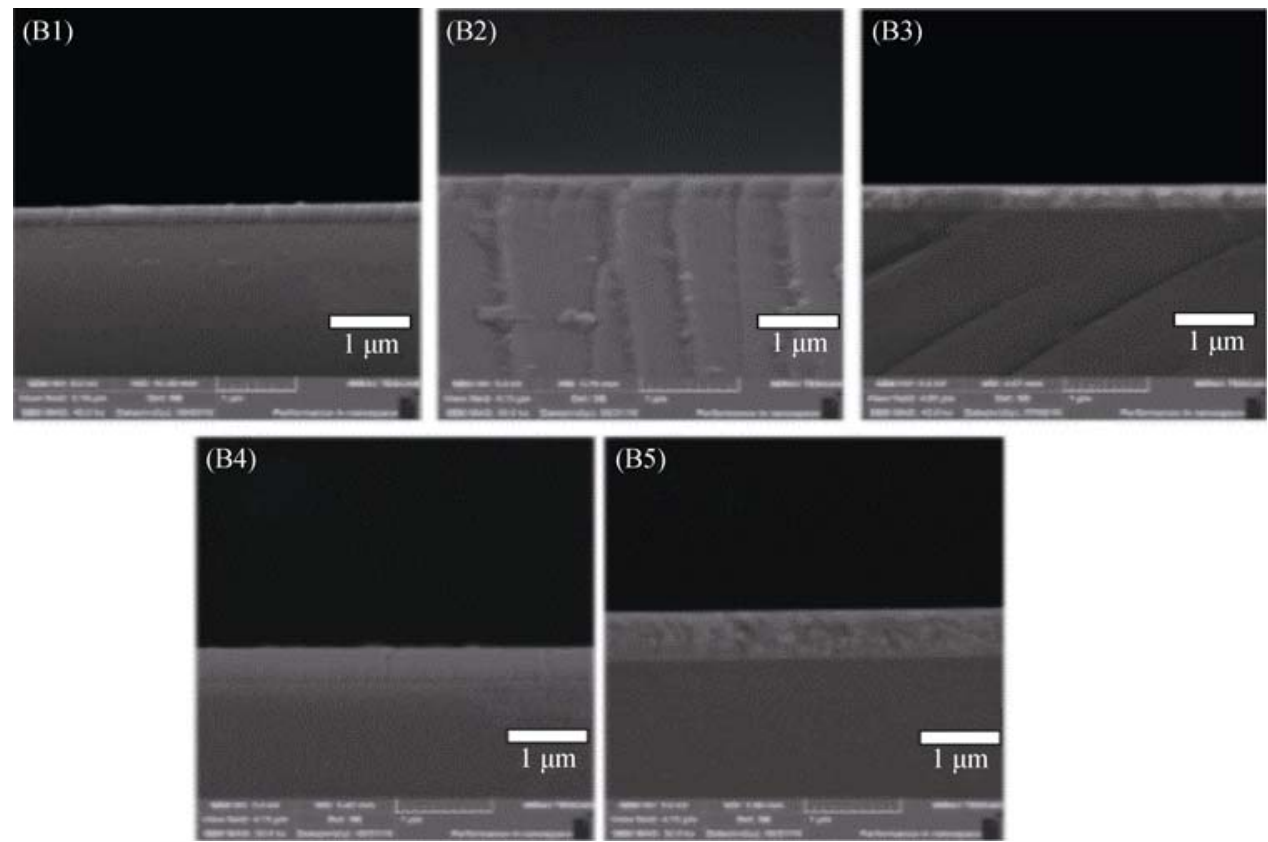

图 7 不同溅射时间制备的 $\mathrm{CeO}_{2} / \mathrm{TiO}_{2}$ 薄膜的 SEM 照片

Fig.7 SEM images of $\mathrm{CeO}_{2} / \mathrm{TiO}_{2}$ thin films sputtered for different time 


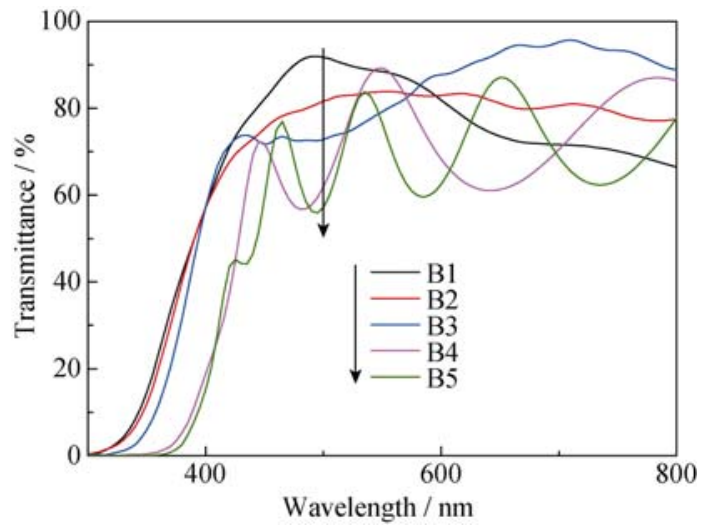

图 8 溅射时间对 $\mathrm{CeO}_{2} / \mathrm{TiO}_{2}$ 薄膜透过率的影响

Fig. 8 Influence on sputtering times on the transmittance of $\mathrm{CeO}_{2} / \mathrm{TiO}_{2}$ thin films

表 3 表面多孔镀膜玻璃与原片镀膜玻璃的透过率

Table 3 Transmittances of the $\mathrm{CeO}_{2} / \mathrm{TiO}_{2}$ coated honeycomb surface glass and raw glass

\begin{tabular}{cccc}
\hline Samples & $\begin{array}{c}\text { Transmittance } \\
\text { of visible/\% }\end{array}$ & $\begin{array}{c}\text { UV cut-off } \\
\text { ratio/\% }\end{array}$ & $\begin{array}{c}\text { Thickness of } \\
\text { the film } / \mathrm{nm}\end{array}$ \\
\hline $\begin{array}{c}\text { Coated raw glass } \\
\text { Coated honeycomb } \\
\text { surface glass }\end{array}$ & 79.8 & 99.1 & 435 \\
\hline
\end{tabular}

合力为 $(9.58 \pm 0.73) \mathrm{mN}$, 而图 9(b)表面多孔玻璃上的 膜基结合力提高了两倍，达(21.32 \pm 0.86$) \mathrm{mN}$ ，说明 玻璃表面的孔结构可以较好地提高薄膜与基板之间 的结合力 ${ }^{[27]}$ 。溅射镀膜过程中，微小的颗粒会溅射
到孔结构中，使薄膜 “嵌入”到基板里面，这种 “嵌 入” 提高了膜与基板之间的接触面积，从而提高了 薄膜在玻璃表面上的结合力 ${ }^{[28-29]}$ 。

\section{3 结论}

采用磁控溅射在玻璃表面镀 $\mathrm{CeO}_{2} / \mathrm{TiO}_{2}$ 膜, 以玻 璃原片为基板优化了溅射功率及镀膜时间，随着 $\mathrm{CeO}_{2}$ 功率的提高, 可见光区域的透过率逐渐降低, 紫外区域的截止率先升高后降低, 当 $\mathrm{Ce} / \mathrm{Ti}$ 原子比为 $50 \%$ 时，紫外截止效果相对较佳。延长镀膜时间会 显著增大膜厚，紫外截止效率会提高，但可见光透 过率下降明显。综合考虑可见光平均透过率和紫外 光的截止率，镀膜时间为 $40 \mathrm{~min}$ (即膜厚为 450 nm) 为佳。

通过采用反胶束微球化学刻蚀制备表面多孔玻 璃，可见光平均透过率提高了 $7 \%$ 。在前述较佳镀膜 工艺的基础上，以所制备的表面多孔玻璃为基板镀 制 $\mathrm{CeO}_{2} / \mathrm{TiO}_{2}$ 膜，当紫外截止率接近 $100 \%$ 时，可见 光透过率达到 85\%，而相同条件下，玻璃原片镀膜后， 可见光透过率只有 $80 \%$ 。此外，玻璃表面上的孔结构 也提高了薄膜与基板的接触面积，膜基结合力提高 2 倍左右。因此，以具有减反增透效果的表面多孔玻璃 为基板镀制功能膜，对提高可见光透过率与增强膜 基结合力具有潜在的应用价值。
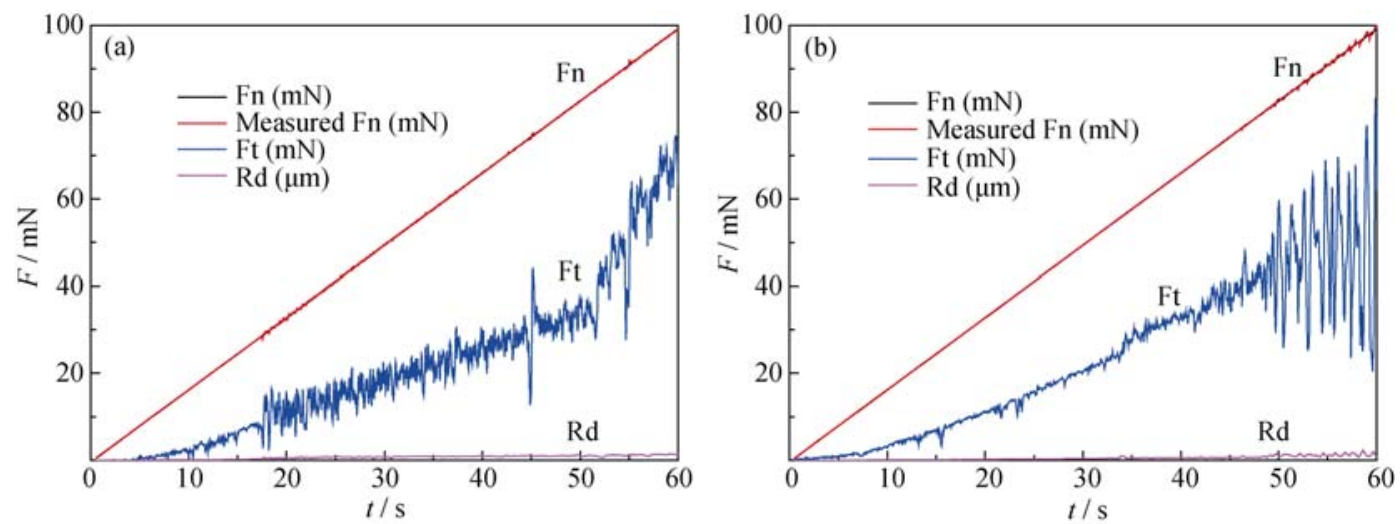

图 9 (a)玻璃原片和(b)表面多孔玻璃基板上薄膜的结合力测试

Fig. 9 Cohesion results of the thin films on (a) the raw glass and (b) the honeycomb surface glass substrates by scratching test

\section{参考文献:}

[1] ZHOU XUEDONG, NI JIAMIAO, ZHAO XIUJIAN, et al. Preparation of $\mathrm{CeO}_{2}-\mathrm{TiO}_{2}$ thin films for $\mathrm{UV}$ absorbing by $\mathrm{RF}$ sputtering. Journal of Wuhan University of Technology, 2006, 28(1): 7-14.

[2] YU XIAOLI, CAO HONGZHANG, ZHANG YUXI, et al. Devel- opment of UV-shielding properties of $\mathrm{CeO}_{2}$. Chinese Rare Earths, 2013, 34(4): 80-84.

[3] TIAN XUECHUN, DONG MENGMENG, XIE HOULI. Application of glass film in energy-saving building. New Building Materials, 2009, 36(8): 78-79.

[4] SAINZ M A, DURÁN A, NAVARRO J M F. UV highly absorbent 
coatings with $\mathrm{CeO}_{2}$, and $\mathrm{TiO}_{2}$. Journal of Non-Crystalline Solids, 1990, 121(1/2/3): 315-318.

[5] MORIMOTO T, TOMONAGA H, MITANI A. Ultraviolet ray absorbing coatings on glass for automobiles. Thin Solid Films, 1999, 351(1/2): 61-65.

[6] SHAO MINGDI, LI MEI, LIU SHAOGANG, et al. Effects of $\mathrm{CeO}_{2}$ on the properties of heat-absorbing glass. Chinese Rare Earths, 2012, 33(4): 19-22.

[7] DONG YUHONG, ZHAO QINGNAN, WU SHUO, et al. Ultraviolet-shielding and conductive double functional films coated on glass substrate by Sol-Gel process. Journal of Rare Earths, 2010, 28(S1): 446-450.

[8] WU SHUO, ZHAO QINGNAN, MIAO DENGKUI, et al. Synthesis and characterization of Sb-doped $\mathrm{SnO}_{2}-\left(\mathrm{CeO}_{2}-\mathrm{TiO}_{2}\right)$ composite thin films deposited on glass substrates for antistatic electricity and uv-shielding. Journal of Rare Earths, 2010, 28(S1): 189-193.

[9] NI JIAMIAO, ZHAO QINGNAN, WANG PENG, et al. Double functional films with UV absorption and transparent conduction deposited on glass by radio-frequency magnetron sputtering. Journal of the Chinese Ceramic Society, 2006, 34(10): 1182-1186.

[10] DU Y, HE H, JIN Y, et al. Optical model of porous glasses using genetic algorithms. Optik-International Journal for Light and Electron Optics, 2013, 124(15): 2093-2096.

[11] FUJIMA T, FUTAKUCHI E, TOMITA T, et al. Hierarchical nanoporous glass with anti-reflectivity and super-hydrophilicity by one-pot etching. Langmuir, 2014, 30(48): 14494-14497.

[12] MENG XIANGMAN, WANG LIANG, CHEN YU, et al. Influence of sodium hydroxide solution on the optical and wetting properties of borate silicate glasses. Journal of Beijing University of Technology, 2015, 41(12): 1911-1914.

[13] 经 验. 减反射玻璃的研制. 北京: 北京工业大学硕士学位论文, 2007.

[14] 高 倩. 大面积氧化物薄膜材料的微纳米结构可控制备与性能 调控技术. 浙江: 浙江大学博士学位论文, 2014.

[15] WANG Z, LIU X, LIU W, et al. Latest development of thin film synthesis technology: reactive magnetron sputtering. Journal of Vacuum Science \& Technology, 2013, 33(12): 1229-1236.

[16] LIU HONGYAN, YAN YUE, WANG YONGLIN, et al. Recent progress in study of transparent conducting oxide films. Journal of Aeronautical Materials, 2015, 35(4): 63-82.

[17] PENG SHOU, JIANG JIWEN, LI GANG, et al. Effect of DC magnetron sputtering process on optical and electrical properties of ITO thin films. Journal of the Chinese Ceramic Society, 2016, 44(7): 987-994

[18] ŠKODA M, CABALA M, CHÁB V, et al. Sn interaction with the $\mathrm{CeO}_{2}(111)$ system: Bimetallic bonding and ceria reduction. Applied Surface Science, 2008, 254(14): 4375-4379.

[19] TAYLOR G. Disintegration of water drops in an electric field. Royal Society of London Proceedings, 1964, 280(1382): 383-397.

[20] CHEN L, LI J, GE M. Promotional effect of Ce-doped $\mathrm{V}_{2} \mathrm{O}_{5}-\mathrm{WO}_{3} / \mathrm{TiO}_{2}$ with low vanadium loadings for selective catalytic reduction of $\mathrm{NO}_{x}$ by $\mathrm{NH}_{3}$. Journal of Physical Chemistry C, 2009, 113(50): 21177-21184.

[21] WU Z, JIN R, LIU Y, et al. Ceria modified $\mathrm{MnO}_{x} / \mathrm{TiO}_{2}$, as a superior catalyst for $\mathrm{NO}$ reduction with $\mathrm{NH}_{3}$, at low-temperature. $\mathrm{Ca}$ talysis Communications, 2008, 9(13): 2217-2220.

[22] WEI F, LIANGSUO N I. Photocatalytic performance and doping mechanism of B-S Co-doped $\mathrm{TiO}_{2}$. Chinese Journal of Catalysis, 2007, 28(10): 905-909.

[23] LIU B, ZHAO X, ZHANG N, et al. Photocatalytic mechanism of $\mathrm{TiO}_{2}-\mathrm{CeO}_{2}$, films prepared by magnetron sputtering under UV and visible light. Surface Science, 2005, 595(1): 203-211.

[24] REDDY B M, KHAN A. Nanosized $\mathrm{CeO}_{2}-\mathrm{SiO}_{2}, \mathrm{CeO}_{2}-\mathrm{TiO}_{2}$, and $\mathrm{CeO}_{2}-\mathrm{ZrO}_{2}$ mixed oxides: influence of supporting oxide on thermal stability and oxygen storage properties of ceria. Catalysis Surveys from Asia, 2005, 9(3): 155-171.

[25] BHARDWAJ N, KUNDU S C. Electrospinning: a fascinating fiber fabrication technique. Biotechnology Advances, 2010, 28(3): 325347.

[26] PURANS J, AZENS A, GRANQVIST C G. X-ray absorption study of Ce-Ti oxide films. Electrochimica Acta, 2001, 46(s 13/14): 2055-2058.

[27] BENJAMIN P, WEAVER C. Measurement of adhesion of thin films. Proceedings of the Royal Society. A Mathematical Physical \& Engineering Sciences, 1960, 254(1277): 163-176.

[28] LUO ZHIHUA, ZHAO YUTAO, LI SUMIN. Structure and properties of $\mathrm{TiO}_{2}-\mathrm{CeO}_{2}$ thin films prepared by magnetron sputtering on polymer substrate. Materials for Mechanical Engineering, 2007, 31(9): $37-40$.

[29] RANDALL N X, FAVARO G, FRANKEL C H. The effect of intrinsic parameters on the critical load as measured with the scratch test method. Surface \& Coatings Technology, 2001, 137(2): 146-151. 